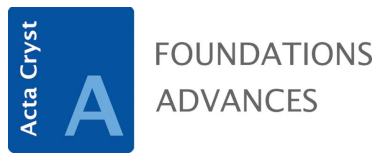

ISSN 2053-2733
Keywords: book review; crystallographic symmetry; International Tables for Crystallography; teaching.

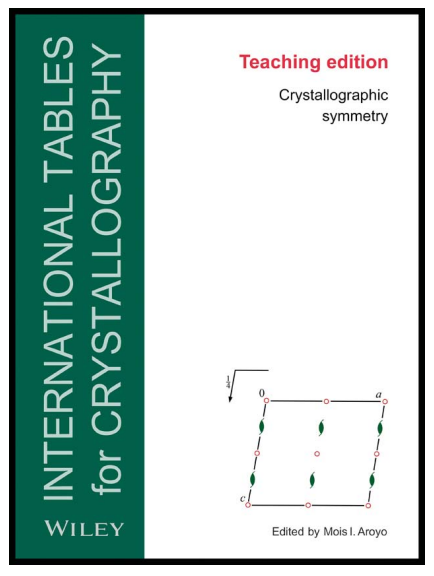

\section{Teaching Edition of International Tables for Crystallography: Crystallographic symmetry. Edited by Mois I. Aroyo. IUCr/Wiley, 2021. Softcover, pp. xii + 236. ISBN 978-0-470-97422-3. Price GBP 29.99.}

\author{
Massimo Nespolo*
}

Université de Lorraine, CNRS, CRM2, Nancy, France. *Correspondence e-mail: massimo.nespolo@univ-lorraine.fr

The Brief Teaching Edition of Volume A of International Tables for Crystallography was first published in 1985 and was meant to serve as a light, easy-to-carry sample of the content of Volume A, containing the diagrams of all of the 17 plane groups as well as 24 space groups covering the seven three-dimensional crystal systems. The pages explaining the symbols used in the diagrams as well as a small subset of the theoretical parts were also included. The usefulness of this edition was essentially in its reduced weight: if one had the complete Volume A, then it would add nothing to what was already in your possession.

Things changed radically this year, with the publication of the new Teaching Edition (the adjective 'Brief' no longer applies), which is no longer a simple selection of some pages from the main volumes (note the plural: the content of three volumes is now presented: A, A1 and E, and even some content related to Volume B is included!) but contains an extensive theoretical part specifically written for teachers of crystallographers, which is however perfectly suited for self-study as well. With this complete reworking of the Teaching Edition we definitely now have a long-overdue complement to the main volumes.

The new Teaching Edition spans 11 chapters divided into two parts: Introduction to crystallographic symmetry (seven chapters) and Crystallographic symmetry data (four chapters). The first part gives the theoretical background needed to understand crystallographic symmetry, i.e. the 'tools' that every crystallographer uses on a daily basis without necessarily mastering them. The presentation is extremely pedagogical and does not require in the reader any prerequisite that goes beyond what an average undergraduate student in any scientific curriculum usually gets (simple matrix algebra is however necessary and, sadly enough, it is no longer present in all curricula). The second part is a step-by-step guide to the use of Volumes A, A1 and E. We discuss below in some detail the content of each chapter.

Part 1. Introduction to crystallographic symmetry. Divided into seven chapters spanning 105 pages, this first part provides the necessary understanding about point, line, plane, space and superiodic groups, with the rigour and precision that one hardly ever finds elsewhere.

Chapter 1.1 (nine pages) is $A$ general introduction to groups. Besides the basic concepts of group theory, available in many textbooks, fundamental concepts like normal subgroups, factor groups and normalizers (too often considered 'beyond the scope' despite the fact that they are essential to understand relations among groups of paramount importance for, e.g., phase transitions) are introduced as well. That all this wealth of information is collected in just nine pages without becoming cryptic for the average reader is definitely impressive.

Chapter 1.2 (seven pages) deals more specifically with Crystallographic symmetry, with particular attention to analysis of the matrix treatment of symmetry operations. The fundamental distinction between geometric element and symmetry element, ignored by almost all textbooks, is duly emphasized.

Chapter 1.3 (15 pages) is A general introduction to space groups. Here the reader finds all they need to understand Bravais lattices, the choice of the unit cells to represent them, 
their metric properties and the classification of space groups. Certainly surprising is to see the crystal systems relegated to the last section (Section 1.3.4.4), among Other classifications of space groups. For a mathematician, this category is probably less fundamental than Bravais classes, presented separately, while for a crystallographer it is the most used classification.

Chapter 1.4 (25 pages) deals with Space groups and their descriptions. A detailed explanation of how HermannMauguin symbols are constructed and concretely used is followed by the presentation of the coordinate triplets, Wyckoff positions and space-group diagrams. The last part is dedicated to sections and projections, whose importance for locating atoms from diffraction data and in the construction of subperiodic groups cannot be overestimated.

Chapter 1.5 (18 pages) is about Transformations of coordinate systems. An updated version of the classical text and tables about the relations between the various types of unit cells is followed by a section about transformations among the various settings of space groups, with the synoptic tables of short, full and extended Hermann-Mauguin symbols up to the orthorhombic crystal system.

Chapter 1.6 (14 pages) is an Introduction to the theory and practice of space-group determination and is more related to Volume B of International Tables. Here the reader learns how to extract symmetry information from the diffraction pattern, with some basic ideas about electron microscopy, in particular convergent beam electron diffraction (although this section is too short to provide more than an appetizer). Four examples of derivation of the space group from the analysis of the diffraction pattern are worked out with enough details to provide the reader with a clear idea of how the procedure works. Particularly welcome is the reintroduction of the diffraction symbol, whose disappearance from Volume A I had criticized in my review (Nespolo, 2017). Its name has been changed from extinction symbol in the previous editions to diffraction symbol, which is definitely more appropriate, extinction being a physical phenomenon completely different to the one leading to the reflection conditions. Note however that the diffraction symbol presented here is a shortened version of the homonymous symbol introduced by Buerger (1942), which also contained the Laue class, and is closer to the aspect introduced by Donnay \& Harker (1940), where asterisks were used instead of hyphens. The symbols used in International Tables are a hybrid of the two and this is the reason why the term extinction symbol was used in the previous editions.

Chapter 1.7 (17 pages) is devoted to Applications of crystallographic symmetry: space-group symmetry relations, subperiodic groups and magnetic symmetry, and is an introduction to the content of Volumes A1 and E that will certainly be perceived as enlightening by the reader who is unfamiliar with these volumes. These topics are of paramount importance for every scientist who goes beyond the "pressbutton/feed-software' blind process, yet they are still today relegated to 'advanced topics' and absent from most textbooks. This chapter will hopefully be the occasion for their democratization.
Part 2. Crystallographic symmetry data. Divided into four chapters spanning 127 pages, this part presents a selection of plane, space and subperiodic group diagrams, enriched by step-by-step user guides.

The first three chapters show how to extract all the necessary information from Volume A (Chapter 2.1: 25 pages of text and 79 pages of symmetry tables), Volume A1 (Chapter 2.2: seven pages of text and five pages of symmetry tables) and Volume E (Chapter 2.3: two pages of text and six pages of symmetry tables).

The last chapter, Chapter 2.4 (two pages) introduces The Symmetry Database, which provides real added value for subscribers to the online series of volumes with respect to the print editions. Indeed, subscribers now have access not only to the electronic (HTML, PDF) versions of the Tables but also to an interactive database which is extremely helpful in the daily work of crystallographers and contains a wealth of information that could not be printed for obvious reason of space.

Because perfection does not belong to this world, a few improvements and corrections, although definitely minor, should be considered for the next printing.

Doubt may rise in the mind of the reader when comparing the following two statements about the rhombohedral lattice.

'For this lattice, the primitive basis $\mathbf{L}_{R}$ consisting of three shortest non-coplanar vectors which are permuted by the threefold rotation is also regarded as a conventional basis' ( $p$. 21); and

'For the space groups within one crystal family the same coordinate system is usually used, which is called the conventional coordinate system (for this crystal family)' (p. 31).

To avoid any misunderstanding it would perhaps be better to emphasize that including the rhombohedral coordinate system among the conventional ones (p. 21) is an exception of practical use with respect to the general rule (p. 31).

A glitch occurs at p. 53, when describing an example of space-group diagram we read that 'the geometric (should be symmetry) element is a diagonal glide plane'.

The transformation of Miller indices under a change of basis (p. 68) is presented in the section 'reflection conditions', but these actually concern Laue indices. While it is true that Miller and Laue indices do transform in the same way, considering the widespread confusion between these two concepts (Nespolo, 2015) a precise distinction would be welcome.

The pleonasm 'holohedry of the lattice' (p. 78) could be avoided. Also, the shortcut 'chirality' for 'chirality sense' (pp. 78 and 86 ) could have been avoided by adopting the more precise term handedness ('same handedness' or 'opposite handedness' is self-evident, whereas 'same chirality' or 'opposite chirality' is puzzling: chirality is the property of being chiral and its opposite is achirality).

The term 'general reflections' (pp. 81 and 85) used in the examples following the section where the classification of reflection conditions (general versus special; integral versus zonal versus serial) might lead to some confusion in the reader about the difference between general reflections and general reflection conditions. A statement drawing the reader's attention could be useful when introducing the examples. 
The citation Glazer et al. (2013) occurring twice at p. 225 is actually from 2014 (the reference is correct, the citation is not).

In conclusion, the Teaching Edition of International Tables for Crystallography is not, and it was not meant to be, a standalone textbook. True beginners who know nothing about crystallographic groups and have never opened International Tables will certainly learn a lot from this book, but will nevertheless need to go for a more basic textbook in order to get an introduction to the subject from scratch. But anybody who has used International Tables, possibly scratching their head over diagrams, trees and coordinate triplets, will find here all the necessary information, provided step-by-step, to finally get the most out of the treasure trove of information collected in International Tables. With a more than affordable price, you have no excuse not to place your order as soon as you reach the period (meaning full stop, not translation!) at the end of this sentence.

\section{References}

Buerger, M. J. (1942). X-ray Crystallography. New York: Wiley. Donnay, J. D. H. \& Harker, D. (1940). Naturaliste Can. 67, 33-160. Nespolo, M. (2015). J. Appl. Cryst. 48, 1290-1298.

Nespolo, M. (2017). Acta Cryst. A73, 274-276. 